Oil sprayed with an atomizer into one nostril returns with almost undiminished volume through the other if it does not meet with an obstruction. Bosworth advises this method for confirming the diagnosis of adenoid growths. Palpation with the index finger introduced gently behind the soft palate is a quick and valuable way to examine the nasopharynx, even if it is not pleasant nor elegant.

The question when lymphatic glands are pathologically enlarged is usually answered without hesitation. As even the most superficial ones can not be felt through the skin when they are in normal condition, it seems reasonable to pronounce tonsillar tissue pathological when it is plainly visible on macroscopic examination.

The differential diagnosis should be quite clear in the vast majority of cases; as retropharyngeal neoplasms and nasal growths extending into the nasopharynx are exceedingly rare in patients between the ages of 5 and 15, when adenoid growths are most prevalent and troublesome. Palpation would, generally, when the patient is well anesthetized, enable one to decide between uncomplicated adenoid vegetations, on the one hand, and nasopharyngeal sarcoma, or fibrosarcoma, or unusual prominence of one of the retropharyngeal lymphatic glands, on the other. An appeal to the microscope might, however, be necessary if the tonsil should be invaded by malignant or tuberculous disease.

\section{IODIN USED HYPODERMICALLY IN THE TREATMEN'T OF PULMONARY TUBERCULOSIS.}

ALFRED CARENO CROFTAN, A.M., M.D.

Formerly Assistant Professor of General Diagnosis and Clinical Instructor of Diseases of the Chest, College of Physicians and Surgeons, Chicago.

PASADENA, CAJ.

The action of iodin on the healthy body has been exhaustively studied; ideal conditions, we may say, were naturally created for such investigation. It seems that the water, soil and air, and consequently the flora of Geneva, Switzerland, are singularly free from iodin; the inhabitants of that region, therefore, constitute a virgin soil for the drug and display a remarkable susceptibility to its action.

Coindet, of Geneva, first described the physiologic effects of the drug; he was led to his studies on iodin while investigating the curative effects of sponge-preparations on goiter-bronchocele-which occurs with such frequency in Geneva; he suspected that iodin was the active remedial principle in the sponge and the other marine products that had for generations been successfully employed for the cure of this affliction. Rilliet, also of Geneva, continued these investigations and in a remarkable "Memoir" presented to the French Academy of Medicine, in 1860, first described iodism and introduced the drug into practice. Trousseau reports on this "Memoir" in the 25th Bulletin of the French Academy. From the reports of these two investigators, that have since been repeatedly corroborated and amplified by reliable authorities-Binz, Niemeyer, Kaemmerer, Sée, Stillé-we glean the following information in regard to the pathogenesy of iodin.

Iodin is peculiarly a drug against which different subjects show marked idiosyncrasies; some penple can take large doses with impunity, others are speedily and violently affected by almost incredibly small doses. Thus Trousseau reports that the Geneva goiters are cured with doses of iodid of potash of less than a milligram, the sponge preparations necessarily contain most minute quantities; in certain sensitive subjects, Rilliet reports "grave accidents" from using quantities of table-salt that contained one part of iodin to 10,000 parts of the salt. He even saw iodism develop in these people from a sojourn at the seashore where, it is estimated, they inhaled from a fiftieth to a tenth of a milligram a day. Cod-liver oil contains about one part of iodin to 40,000 parts of oil, and it is not impossible that the curative effects that this marine product exercises on certain "strumous" diseases and the benefits that are claimed for it in tuberculosis may be attributed to the presence of even so minute a quantity of iodin.

The chief symptoms observed are emaciation, usually accompanied by profuse sweats, some pyrexia and an accelerated pulse; a peculiar psychical depression develops a form of hypochondriasis, "anxietas."

A large field of action is the mucous lining of the respiratory tract; when the system becomes saturated with iodin, almost any of the manifestations of inflammation of these tissues may supervene, coryza, angina, laryngitis "with hoarseness, aphonia and chronic inflammation, even simulating laryngeal phthisis;" the lungs show" "congestive oppression, and hemoptysis may occur;" Zeiss 1 reports an "abundant serosity" in the lungs of dogs that had been subjected to subcutaneous injections of iodin and an iodid in solution. The serous membranes, too, become affected and pleuritis has been observed. These effects on the respiratory passages, it must clearly be understood, are remote effects of the drug and follow the administration of iodin wherever in the body it may have been introduced; they must not be confounded with its local irritative action following inhalation or topical application.

The lacteals and the lymphatic system are probably the main sphere of action; iodin acts as an irritant, and possibly this explains the emaciation, inasmuch as the chyle is absorbed more slowly, is insufficiently elaborated by the mesenteric glands, and the absorption of fats is, as a consequence, reduced. Hence, probably, too, the profound remedial action of iodin on bronchocele, lymphatitis-scrofulosis, strumous diathesis-and tabes mesenterica, in all of which affictions the lymphatic and lacteal systems are inclined to hyperplasia, with resulting sluggish function, etc.

If we study the symptomatology of incipient tuberculosis we find that essentially the same tissues are stimulated to a reactive display of energy by the tubercle virus as by iodin. Again, we must carefully exclude the local, mechanical, and destructive action of the living parasite, and try to draw into the circle of our considerations only the remote effects that are exercised by the products of microbian life near the focus of infection and in distant parts of the body.

Locally, we have that "liquefaction of tissue" that is so commonly attributed to the action of iodin; remotely, the specific selective affinity for the respiratory mucosa, the serous membranes, the lympho-lactea] system; we have the emaciation accompanied by pyrexia, tachycardia and sweats; probably the initial psychosis so often observed is the same.

In the light of our theoretic beliefs the administration of iodin should act curatively in pulmonary tuberculosis; the virus of tuberculosis, entering the blood at first in minute quantities, produces certain slight reactive phenomena from the

1. La Clinique, Feb., 1900. 
cells that it is selectively attracted to; this reaction may or may not become subjectively and objectively perceptible; that will depend on its intensity, the character of the reactive symptoms and our facilities for recognizing these symptoms with the rather crude clinical means at our disposal and the uncertain evidence of our senses. If the stimulation and the resulting reaction are strong enough, then the vis medicatrix can protect the organism unaided; if it is not sufficiently powerful then the organism is in danger, and it is here that a substance that is capable of irritating the same cells that are reacting to the tubercle virus can produce an increased reaction and aid nature.

Finally, if a carefully graded and sufficiently small dose of iodin will exercise a curative effect in tuberculosis, then a large dose should aggravate by overstimulating and consequently exhausting the very cells that are by their reaction counteracting the toxin invasion. Clinical experience bears this out. Knopf speaks of the "congestive and softening" action of the drug in incipient and latent tuberculosis, and condemns the practice of administering iodids for diagnostic purposes in such cases; it increases bronchial secretion and generally accentuates the physical signs. Hughes speaks of "the occasional development of phthisis pulmonalis in iodized patients in whom apparently no previous tendency thereto had existed."

Accurate dosage is essential to the success of the plan of treatment that is being advocated; too large doses of iodin will certainly aggravate, too small doses will be inefficient. A variety of plans were tried to secure accurate dosage of iodin. At first, the administration of iodid of potash by mouth was considered; it was expected that, according to the researches of Binz, the alkaline iodid, when it reached the tissues, would in the presence of protoplasm and $\mathrm{CO}_{2}$ be decomposed and free iodin be liberated; this actually occurs, but only a small portion of the iodid of potassium is dissociated in this way and a large proportion is voided in the urine and wasted. On the other hand, Sée calls attention to the fact that iodin, when given by mouth, necessarily unites with the sodium in the stomach contents and is consequently absorbed as alkaline iodid; this entering the tissues is again partly decomposed and partly wasted; accurate dosage of iodin by mouth, either as iodin or in the form of its alkaline salts, is, therefore, impossible.

Administration by inunction in an oily menstruum was considered; europhen and the other non-irritating iodin compounds recently brought before the profession lend themselves very readily to such use, but accurate or even approximately accurate dosage is, of course, impractical. Hypodermic administration of iodin seemed the only reliable plan, and here the irritative properties of the drug seemed to prohibit its use; at this juncture our attention was called to a paper by Klingmueller, ${ }^{2}$ in which he reports the results of his investigations with an iodin preparation called iodipin, which is an iodin addition product of sesame oil, a yellow fluid of oleaginous taste, 10 per cent. iodin. Klingmueller reports as follows: "It was first found by experiments on animals that the drug employed hypodermically was not toxic; thirty-six patients were treated and received 220 injections of a 10 per cent. preparation. No unpleasant effects were observed even when 20 c.c., the equivalent of 30 grains of iodin, were injected daily. . . . Subsequently additional injections of a 25 per cent. preparation were made with

2 Berlin klin. Woch., June 19, 1899. equally satisfactory results. Not only was the iodin deposited in the subcutaneous tissues slowly absorbed and distributed, but all of it was necessarily taken up and rendered active. Iodin appeared in the urine in from three to five days after the treatment was begun and its excretion continued for several weeks, while in the other preparations the iodin appears earlier and the period of elimination is much shorter.

The subcutaneous method of injection of iodipin has the further advantage of being painless, convenient and cheap. . . . The specific action of iodin was manifested after subcutaneous injections of iodin in the same degree as when other iodin preparations, and especially iodipin, were injected by mouth."

Before attempting the hypodermic administration of iodipin in human subjects, the advisability of preliminary animal experiments was considered; however, it is well-nigh impossible to experimentally simulate tubercular invasion as it actually occurs; instead of gradually gaining a foothold in some weakened spot of a bronchus or bronchiolus, the bacillus in laboratory tuberculosis is injected in enormous quantities directly into vulnerable tissues; its virulence is great; the animal's natural means of defense are speedily overcome and it succumbs in every instance. Furthermore, we know that iodipin injected into a non-tubercular subject can do no harm and that, if the dose be carefully regulated, only beneficial effects can be expected in a tubercular case. Animal experiments were, therefore, waived as unnecessary and the administration of iodipin tried directly on patients in whom the diagnosis of tuberculosis of the lungs had been positively made.

Iodipin was employed in the form of the 10 per cent. preparation. The injections were made into the subcutaneous tissues between the skin and the muscle, and preferably in the gluteal and interscapular regions. No discomfort of any kind was ever caused, no inflammatory reaction observed at the site of the injections, though some patients received daily injections for a period of three or four months.

Beginning with one drop of iodipin, which, to give the necessary bulk for hypodermic administration, was dissolved in half a dran or so of sterilized oil, the injections were gradually increased, one drop being added to the dose each day. The dosage was regulated by the symptoms; as soon as an improvement became apparent, the dose exhibited at the time was continued for a period of thirty to sixty days. Much will depend on the individual idiosyncrasies of the patient and the stage of the disease. In some cases the quantity that acted beneficially for a time seemed to grow insufficient and the dose was again increased drop by drop; more than 60 minims a day have so far never been given. Bad effects from this mode of administration were never observed. As 100 drops of the 10 per cent. iodipin weigh $2.5 \mathrm{gr}$., and consequently represent $0.25 \mathrm{gr}$. of iodin, each drop of iodipin administered represents only $0.0025 \mathrm{gr}$. of iodin, or about 0.04 grains.

The object of giving the drug in gradually increasing doses is apparent from our theoretic postulates; it is twofold: 1, to avoid the exhibition of too large a dose, the aggravating effects of which are recognized; 2 , in order to create a full artificial immunity against the drug; by employing this plan much larger doses can be administered finally with impunity and more decided reactive effects produced in the end.

The results obtained so far by this plan of treatment have been uniformly good; in a few cases amelioration of symptoms was marked from the beginning; appe- 
tite improved, the cough, the night sweats grew less stvere, the patients gained in weight and improved in spirits. The physical signs were modified and seemed to show that the process was at least being held in check and rendered latent; in 2 out of my 27 cases a recurrence of symptoms occurred at the expiration of three and five months, respectively; these patients underwent a second course of treatment with good result. It is altogether too early to make any statements in regard to a cure.

Iodipin injections were tried only on carefully selected cases. Where great destruction of lung tissue had occurred, when the patients were devitalized by long-lasting mixed infection, in other words, in the so-called tertiary stage of the disease, the treatment was not instituted.

Twenty-seven cases have so far been treated with good results; 19 were cases of incipient tuberculosis with only circumscribed areas of infection in one or the other of both apices; in the other 8 , larger areas in one or both lungs were involved; in 7 of the 27 cases, streptococcus infection co-existed; the remaining 20 were simple infections. Detailed reports of my crices are reserved for future publication, when more clinical material shall have been adduced and the evidence in support of our theories and our practice shall have grown more numerous and more positive.

The results obtained so far are not conclusive; they are only suggestive, and final judgment on the efficacy of this method of treatment must be suspended. The results, it is true, appear to be sufficiently striking to warrant an optimistic view. The number of cases treated, however, is small and the subjects were carefully selected; in addition to the iodipin injections the patients enjoyed the benefits of all the hygienic and dietetic measures suitable to each case and the numerous advantages incident to outdoor life in the warm and equable climate of Southern California.

Notwithstanding all this, it seems more than coincidence that of the 27 cases on record all improved under iodipin injections; if nothing else, these findings should encourage to further investigation and the collation of further clinical evidence.

In conclusion, we make an earnest plea for the early recognition of pulmonary tuberculosis; the sooner the disease is recognized and treatment begun, the better the prognosis. Our clinical experience in the past leads us to join the ranks of those who consider pulmonary tuberculosis, in its incipiency and before it has become a mixed infection, one of the most easily curable of bacterial diseases.

\section{HERNIA OR DIVERTICULUM OF THE CHORION.}

I. H. LAIDLEY, M.D. ST. LOUIS.

Cysts of the placenta have been reported by Millet and others, supposed to have developed from the exudation of blood forming a clot separating the chorion, usually of small size, and in some cases more than one is noted. Ercolani reported two cases; in one the entire fetal surface of the placenta was sown with round tumors covered by chorion - the largest being about the size of a cherry. Some have been opened and the chorionic wall torn, showing a solid material filling the depth of the cyst; others were more solid, and were filled with coagulated fibrin, in which rounded masses of granular hematin could be seen.
Ercolani proved that the interior wall was formed by the chorion, which covered the whole bloody mass, of which half projected above the placental surface, while half dipped into the placental tissue, and lay in immediate contact with the effused blood. The term cyst is therefore inexact. At the placental depth of the tumors, the villosities, more fibrinous than usual, formed a compact layer, certain spots in which turned out to be cells of the serotina. Small irregular calcareous concretions were scattered through the mass.

Bustamente describes a kind of cyst which is sometimes found on the fetal surface of the placenta, of a regularly rounded or elongated shape and varying in size from $2 / 5$ of an inch to 2 or $22 / 5$ inches. They are placed below the chorion and amnion, which form their superficial fetal boundary, being limited below by

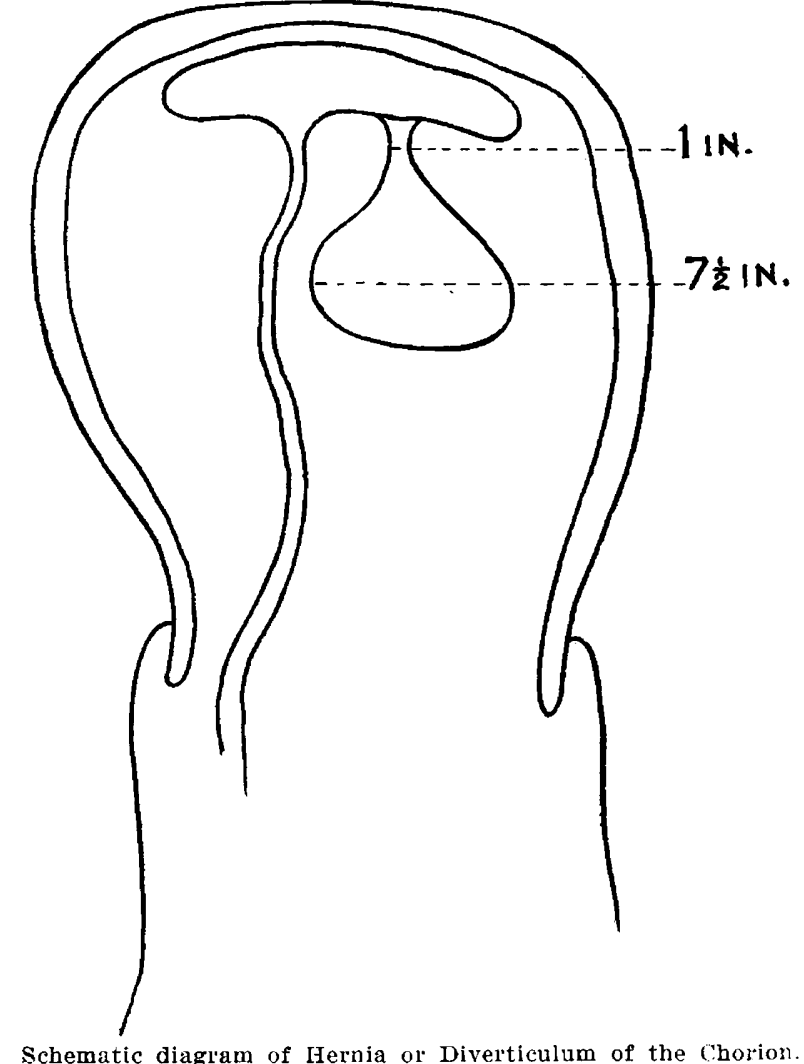

the placental tissue itself. The contents of these cysts were solid and liquid.

A. Daugan reports a case of an oval tumor $42 / 5$ inches long by $31 / 5$ inches broad and covered by the membranes, which are partly detached from its surface. Several large venous and arterial branches of the umbilical vessels run over its surface and penetrate its substance to the center. Divided longitudinally, the tumor appears to be composed of intimately adherent lobes, some being of a dead-white and others of a paleor deep-rose tint. Its tissue is homogeneous.

The case which I present has the following history: Mrs. I., age 32 , was confined at the seventh month, being her second pregnancy, on August 5. She was in labor about six hours. All that time the os was fully dilated, without any pain, until a few minutes before her delivery, which was normal; twenty minutes afterward a tumor-like body $\gamma$ inches in diameter was expelled, proving to be a sac containing an amniotic fluid, with coagulated blood. It was attached to the placental covering; 1 inch to the left of the funis, making a pedicle $1 / 2$ inch in diameter, made up of chorionic 